when carefully searched for in all but one patient. ANA and RF were found in 13 of 17 and "scleroderma pattern" was noted in 9 of 13 patients on nailfold capillaroscopy. Taut skin developed later in $57 \%$ of pts following extracutaneous presentation by 1 month to 7 yrs (mean 2.3 yrs).

Conclusion Clues to the diagnosis of ssSSc can be classified as: 1) background conditions - unclassified system disorder, Raynaud's, polyarthritis 2) evolving visceropathy of obscure provenience - dysphagia, malabsoption, pulmonary fibrosis, heart failure, nephropathy 3) corroborative laboratory and clinical features - hyper/hypopigmentation, telangiectasis, calcinosis, typical nailfold capillaroscopy 4) confirmatory evidence - visceral biopsy, appearance of taut skin.

\section{FRI0206 PLASMA HOMOCYSTEINE LEVELS IN PATIENTS WITH SYSTEMIC SCLEROSIS}

${ }^{1} \mathrm{~T}$ Pirildar, ${ }^{2} \mathrm{~N}$ Turgan, ${ }^{1} \mathrm{E}$ Tunc, ${ }^{1} \mathrm{~T}$ Turk, ${ }^{2} \mathrm{D}$ Ozmen, ${ }^{1} \mathrm{G}$ Keser, ${ }^{2} \mathrm{O}$ Bayindir, ${ }^{1} E$ Doganavsargil. ${ }^{1}$ Rheumatology; ${ }^{2}$ Clinical Biochemistry, Ege University, Izmir, Turkey

10.1136/annrheumdis-2001.287

\section{Background}

Objectives Homocysteine is a nonessential amino acid containing sulfur. Homocysteine has a toxic effect on the endothelium suggesting that it may take part in the etiopathogenesis of the diseases with vascular impairment. Since complex etiopathogenesis of systemic sclerosis (SSc) involves endothelial injury and activation, elevated plasma homocysteine levels might be expected in this disease. Standing from this point, we investigated plasma homocysteine levels in patients with limited (ISSc) and diffuse (dSSc) systemic sclerosis and in healthy controls.

Methods Serum samples were collected from 59 (31 limited and 28 diffuse; F/M:56/3 male) patients with systemic sclerosis attending Ege University Rheumatology department, and 27 age and sex matched healthy controls were included in the study. Homocyteine was measured by high-performance liquid chromotography. Hyperhomocyteinemia may occur due to diabetes mellitus, hyperlipidemia and vitamin B12, folic acid deficiencies. Therefore, patients with these conditions excluded from the study.

Results Statistical analysis was performed using one way analysis of varience test. All data are presented as mean $=S D$. A p value $<0.05$ was accepted as statistically significant.

Plasma homocyteine levels in patients with diffuse SSc (16.31 $\pm 5.916 \mathrm{mmol} / \mathrm{L})$ and limited SSc $(13.27 \pm 8.352 \mathrm{mmol} / \mathrm{L})$ were significantly higher than healthy controls $(6.34 \pm 1.540$ $\mathrm{mmol} / \mathrm{L}$ ). When we compared the two subgroups of SSc, plasma homocystein levels in diffuse SSc, was significantly higher than limited SSc $(\mathrm{F}=19.22 \mathrm{p}<0.001)$.

Conclusion Numerous clinical studies have been published on the association between homocysteine and vascular diseases. It has been shown to be cytotoxic to endothelium, to induce a vascular-endothelial cell activator, and to promote smooth muscle cell proliferation. Our finding of

Significantly higher plasma homocysteine levels in the both subgroups of SSc implicate the contribution of hyperhomocysteinemia to endothelial damage in this disease.

\section{FRI0207 ASSESSMENT OF TRIO AS THE CANDIDATE GENE FOR FAMILIAL CHONDROCALCINOSIS CCAL2}

${ }^{1}$ A Pendleton, ${ }^{1} \mathrm{GD}$ Wright, ${ }^{2} \mathrm{MD}$ Doherty, ${ }^{3} \mathrm{R}$ Shiang, ${ }^{3} \mathrm{AE}$ Hughes. ${ }^{1}$ Rheumatology, Musgrave Park, Belfast, UK; ${ }^{2}$ Academic Rheumatology, University of Nottingham, Nottingham, UK; ${ }^{3}$ Medical Genetics, Queens University Belfast, Belfast, UK

\subsection{6/annrheumdis-2001.288}

Background Chondrocalcinosis is usually caused by the deposition of calcium pyrophosphate crystals on articular cartilage. There is a clear relationship between this crystal deposition and excess extracellular triphosphate and pyrophosphate. TRIO, a novel multidomain transmembrane protein which promotes the exchange of guanine diphosphate for guanine triphosphate in the extracellular space is an excellent positional candidate gene for CCAL2.

Objectives

Methods A positional candidate gene strategy was employed as a valid approach to identify the putative disease locus for CCAL2. The Online Mendelian Inheritance in Man OMIM cytogenetic map was used to identify TRIO as a possible candidate gene within the critical region on chromosome $5 \mathrm{p}$. Mutation analysis required the design of PCR primers to amplify the coding DNA from human genomic DNA in affected individuals. The coding DNA sequence for TRIO was known ${ }^{1}$ but information on the intron-exon splicing sequences was unavailable. Sotgun sequence obtained from a P1-derived artificial chromosome (PAC) 514p4 from a PAC contig of the critical region had shown homology with TRIO using the homology search algorithms BLASTN. The intron-exon junctions were identified from this PAC and other adjacent PACs on the contig, using a combination of vectorette PCR and the Genome Priming System (New England Biolabs inc) followed by Big Dye Terminator sequencing on an $377 \mathrm{ABI}$ genetic analyser.

Results From the TRIO coding DNA sequence of 9 kilobases over 55 exons were identified. Both of the functional guanine exchange factor (GEF) domains (18 exons) have been fully sequenced but no mutations have yet been identified in affected members from two large families.

Conclusion TRIO is a novel multidomain transmembrane protein which is localised within the CCAL2 region on chromosome $5 \mathrm{p}$. It is likely to influence the availability of extracellular triphophate and thus pyrophosphate through the function of the active GEF domains. This gene however has no mutations within the coding regions for both the GEF domains in two large kindreds with familial chondrocalcinosis.

\section{REFERENCE}

1 Debant A, Serra-Pages C, Seipel K, O'Brien S, Tang M, Park S-H, et al. The multidomain protein Trio binds the LAR transmembrane tyrosine phosphatase, contains a protein kinase domain, and has a separate rac-specific and rho-specific guanine nucleotide exchange factor domains. Proc Natl Acad Sci USA 1996;93:5466-71

\section{FRI0208 COEXPRESSION OF TH1 AND TH2-ASSOCIATED CHEMOKINE RECEPTORS IN T CELLS INFILTRATING EARLY FIBROTIC SKIN IN A SLE PATIENT}

${ }^{1} \mathrm{C}$ Chizzolini, ${ }^{2} \mathrm{M}$ Hess, ${ }^{2} \mathrm{MG}$ Uguccioni, ${ }^{2} \mathrm{M}$ Baggiolini, ${ }^{1} \mathrm{JM}$ Dayer, ${ }^{2} \mathrm{P}$ Loetscher. ${ }^{1}$ Division of Immunology and Allergy, Geneva University Hospital, Geneva, Switzerland; ${ }^{2}$ Theodor Kocher Institute, Bern University, Bern, Switzerland

10.1136/annrheumdis-2001.289

Background Fibrotic skin changes in systemic sclerosis (SSc) and allied conditions characteristically are preceded by an 
inflammatory infiltrate rich in T cells. It is not known whether recruited $\mathrm{T}$ cells induce fibrosis or react to developing fibrosis. Objectives Chemokines (CC) are involved in cell recruitment into inflammatory sites. Our aim was to characterise the CC receptor $(\mathrm{R})$ usage as well as the effector function of T cells infiltrating a developing fibrotic skin lesion in a SLE patient.

Methods T cells from a lesional skin biopsy were grown in vitro in the presence of IL-2. TcR, accessory molecules, and CCR expression were analysed by flow cytometry and RNase protection assay. The capacity to release interferon-g (IFN-g) and IL-4 was assessed upon CD3-crosslinking in phenotypically homogeneous $\mathrm{T}$ cell lines or clones. The chemotactic responses to synthetic CC was assessed. Immunohistochemistry was performed on the same tissue.

Results $\mathrm{T}$ cells infiltrating the affected skin were heterogeneous in terms of accessory molecules $\left(\mathrm{CD} 4^{+}, \mathrm{CD} 8^{+}\right.$, and CD4CD8 double negative) as well as of $\operatorname{TcR}(\mathrm{a} / \mathrm{b}$ and $\mathrm{g} / \mathrm{d})$ expression. The majority preferentially produced IFN-g, while a minority produced both IFN-g and IL-4. Preferential expression of type-1related CXCR3 (and response to relevant CC) was observed and confirmed by tissue immunohistochemistry. Interestingly, several T cells coexpressed type 1 and type 2-related CCR and migrated to an equivalent extent in response to specific ligands.

Conclusion The analysis performed on T cells infiltrating a novel fibrotic skin lesion from a SLE patient has revealed heterogeneous expression of CC receptors by $\mathrm{T}$ cells preferentially producing IFN-gamma. Since IFN-gamma exerts a potent inhibitory activity on ECM deposition by fibroblasts, these results hint to a reactive mechanisms aimed to control the extent of fibrosis.

\section{REFERENCE}

1 Chizzolini C, Rezzonico R, Ribbens C, Burger D, Wollheim FA, Dayer J-M. Inhibition of type I collagen production by dermal fibroblasts upon contact with activated T cells. Different sensitivity to inhibition of systemic sclerosis and control fibroblasts. Arthitis Rheum. 1998;41:2039-47

\section{FRI0209 RATE OF CEREBRAL MICROEMBOLI IN APS CAN BE INFLUENCED VIA BLOCKADE OF GPIIB/IIIA PLATELET RECEPTOR WITH TIROFIBAN}

${ }^{1} \mathrm{C}$ Specker, ${ }^{1} \mathrm{~A}$ Perniok, ${ }^{2} \mathrm{~T}$ Menge, ${ }^{2} \mathrm{M}$ Siebler, ${ }^{1} \mathrm{M}$ Schneider. ${ }^{1}$ Department of Rheumatology; ${ }^{2}$ Department of Neurology, Heinrich-Heine-University, Düsseldorf, Germany

\subsection{6/annrheumdis-2001.290}

Background Patients with antiphospholipid syndrome (APS) are prone to thromboembolism. So far, the only predictive parameters for further complications are their number in patient's history and perhaps the titre of aPL. Derived from clinical investigation of stroke and obvious analogies between cerebrovascular ischemia (CVI) in patients with carotid artery disease (CAD) and patients with APS, a novel non-invasive method using transcranial Doppler (TCD) is used for detection of so called 'microembolic signals' (MES) in the cerebral vasculature. In patients with $\mathrm{CAD}$, these 'microemboli' proved to correlate with past and impending symptoms of CVI allowing for therapeutic stratification by their detectability. We found very similar signals in the cerebral bloodstream of APS patients with a history of CVI.

Detectability of MES in APS implies occlusive thromboembolism and not vasculitis as the underlying mechanism at least in arterial cerebrovascular manifestations of this state. However, Doppler signal properties (i.e. the reflected energy) do not allow for direct estimation of the signal's substrate size or composition.

Objectives To further elucidate the pathophysilogical substrate of MES in APS.

Methods Repeated TCD monitoring in a 38 year old female APS patient with ongoing transitory ischaemic attacks and high rate of MES before and after intravenous administration of Tirofiban, a potent, synthetic, highly selective and reversible inhibitor of platelet gpIIb/IIIa receptor which binds fibrinogen on the platelet's surface.

Results Within one hour the rate of MES decreased from 50/ $\mathrm{hr}$ to $<5 / \mathrm{hr}$ on three successive occasions accompanied by cessation of clinical symptoms. Using flowcytometry, platelet-leucocyte aggregates found increased before administration of Tirofiban were also reduced considerably.

Conclusion The finding that MES are influenced by blockade of the platelet gpIIb/IIIa receptor and correlate with platelet-leucocyte aggregates argues for a central role of thrombocytes in APS with CVI. This might favour to look for thrombocyte targeted prophylactic therapy in APS. Whether detection of MES can also serve to monitor such therapies has still to be proved.

\section{REFERENCES}

1 Specker C, Rademacher J, Sohngen D, Sitzer M, Janda I, Siebler M, Steinmetz H, Schneider M. Cerebral microemboli in patients with antiphospholipid syndrome. Lupus 1997;6(8):638-44

2 Specker C, Perniok A, Brauckmann U, Siebler M, Schneider M. Detection of cerebral microemboli in APS - introducing a novel investigation method and implications of analogies with Carotid artery disease. Lupus 1998;7(Suppl 2):75-80

\section{FRI0210 CARDIAC INVOLVEMENT IN SYSTEMIC SCLEROSIS (SSC) - DOPPLER ECHORARDIOGRAPHY STUDIES IN 100 SSC PATIENTS}

AV Volkov, GP Kotelnikova, ES Match. Department of Microcirculation and Inflammation, Institute of Rheumatology of RAMS, Moscow, Russia

\subsection{6/annrheumdis-2001.291}

Background Cardiac involvement is a relatively common occurrence in systemic sclerosis. When this complication is clinically recognisable, the prognosis is usually poor. However, heart abnormalities is frequently asymptomatic and they can only be diagnosed by Doppler- echocardiography.

Objectives The aim of this study is to find out the frequency of different variants of cardiac involvements in SSc pts and connexion with other SSc manifestation.

Methods 100 SSc pts (18 male and 82 women, mean age \pm SD $51,8 \pm 14,1$ years, ranging from 15 to 83 years, mean duration of disease \pm SD 10,11 $\pm 9,22$ years). All of the pts were clinically studied with Doppler Ehocardiography.

Results According to the Doppler Ehocardiography studies cardiac involvement was found in $82 \%$ occurences, and was the most frequent symptoms of internal organ damage. Pericard, myocard and endocard were involved. Pericarditis was found in $30 \%$ pts, higher frequency $(25 \%)$ in limited cutaneous SSc, and correlated with disease duration. Pericardit pts have more frequently interstitial lung disease, pulmonary hypertension. The microcirculation changies such as tendency to decreasing of basal digital blood flow and the reduction of capillar loops density were noted in the pericardit patients. The changes in the size of left ventricular were more frequent in pts with diffuse cutaneous $\mathrm{SSc}$, shorter duration of the disease and artherial hypertension, the latter does not exclude the influence of age factors in evolution of these changes. Diastolic disfunction was found in $25 \%$ 